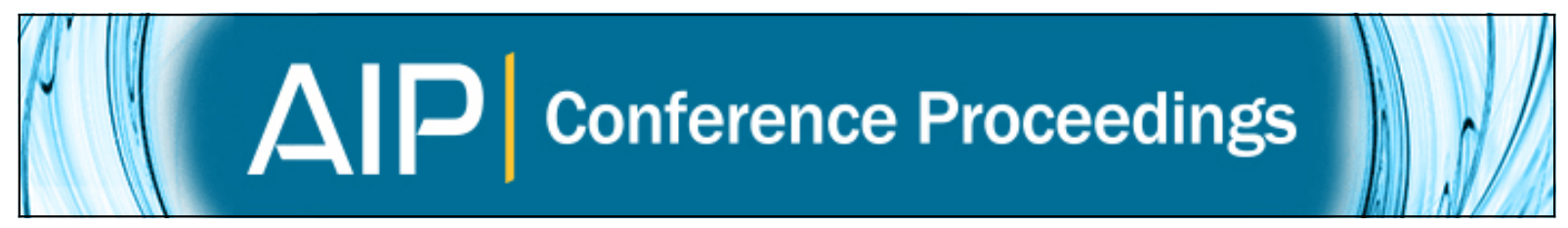

\title{
Numerical simulation of turbulent heat transfer close to the critical point
}

B.J. Boersma, R. Pecnik, H. Nemati, and J. Peeters

Citation: AIP Conference Proceedings 1648, 030006 (2015); doi: 10.1063/1.4912323

View online: http://dx.doi.org/10.1063/1.4912323

View Table of Contents: http://scitation.aip.org/content/aip/proceeding/aipcp/1648?ver=pdfcov

Published by the AIP Publishing

Articles you may be interested in

Direct numerical simulation of turbulent heat transfer in a fluid-porous domain

Phys. Fluids 25, 125110 (2013); 10.1063/1.4851416

Direct numerical simulation of turbulent supercritical flows with heat transfer

Phys. Fluids 17, 105104 (2005); 10.1063/1.2047588

Direct numerical simulation of stagnation region flow and heat transfer with free-stream turbulence

Phys. Fluids 15, 1462 (2003); 10.1063/1.1565332

Numerical simulation of turbulent heat flows

Phys. Fluids 29, 3684 (1986); 10.1063/1.865800

Numerical simulation studies of a turbulent heating experiment

Phys. Fluids 17, 2255 (1974); 10.1063/1.1694700 


\title{
Numerical Simulation of Turbulent Heat Transfer Close to the Critical Point
}

\author{
B.J. Boersma, R. Pecnik, H. Nemati and J. Peeters \\ Department of Process \& Energy, Delft University of Technology, Leeghwaterstraat 39, 2628 CA Delft, The \\ Netherlands
}

\begin{abstract}
In this paper we discuss the effect of sharp property variations on the turbulent heat transfer in fluids close the critical point. The governing equations for this flow regime are discussed, a short description of the numerical tools that have been developed to study these flows is given. Finally, some results for supercritical heat transfer in developing turbulent pipe flow are presented.
\end{abstract}

Keywords: Turbulence, Supercritical flows, Heat transfer, Direct Numerical Simulation

PACS: $47-27$

\section{INTRODUCTION}

Turbulence and turbulence enhanced heat transfer with slowly varying properties as a function of temperature has been studied intensively in the past, e.g. for Helium systems [1]. Recently, pushed by possible technical applications, there is a growing interest in fluid behaviour with strongly varying thermodynamic properties. Here we want to investigate the effect of large changes in material properties on heat transfer in three dimensional turbulent flows for technically relevant fluids, such as water-steam and carbon-dioxide. The large variation of thermodynamic properties near the critical point leads to large root-mean-square density fluctuations (up to $60 \%$ of the local mean density) and buoyancy induced flow phenomena, which affects laminarization, and can lead to deterioration or enhancement of heat transfer. This change in heat transfer is shown in Figure 1, which is taken from [2] where the heat transfer coefficient $h$ for a carbon-dioxide system near the critical point is plotted as a function of the temperature.

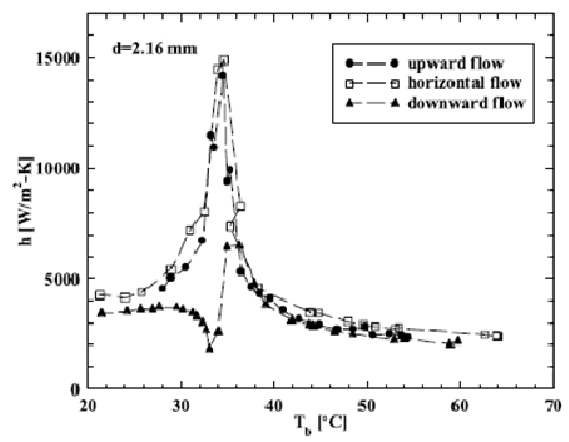

FIGURE 1. The experimentally observed heat transfer coefficient of a turbulent $\mathrm{CO} 2$ flow in a cylindrical pipe, for different orientations of the gravity, as a function of the temperature, from [2]. Note the very strong increase of the heat transfer coefficient in the vicinity of the critical point $\left(\right.$ Tcrit $\left.=31.1^{\circ} \mathrm{C}\right)$.

Depending on the direction of gravity, a large change of the heat transfer coefficient can be observed close to the critical point (Tcrit=31.1C). The large influence of the direction of gravity on the heat transfer coefficient shows how important buoyancy effects are in these flows. Furthermore, it should be stressed that the heat transfer coefficient is an average value: the effects can be much larger locally. To give an idea of the change in magnitude of the material properties of fluids we have plotted the heat capacity, density and dynamic viscosity of water/steam at a constant pressure of $25 \mathrm{MPa}$ (slightly higher than the critical pressure) over the temperature range $300-500^{\circ} \mathrm{C}$. The density and viscosity show moderate changes, while the changes in the heat capacity are extremely large. 


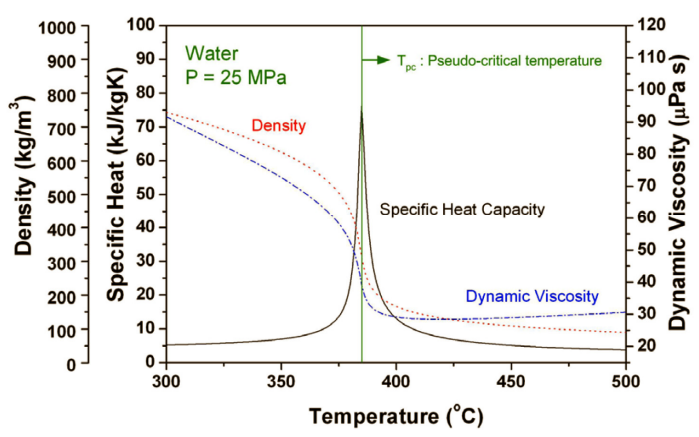

FIGURE 2. The values the dynamic viscosity, density and specific heat capacity of water/steam at a constant pressure of $25 \mathrm{MPa}$ as a function of temperature (from the IAWCS-IF97 formulation [3]). Note that the pseudo critical temperature (the temperature fore with the heat capacity has its highest value) is slightly higher than the critical temperature.

As it can be observed from Figure 2, also physical properties such as the fluid viscosity and density vary considerably. Especially the (expected) local strong increase in turbulent transport could lead to damage of the heat exchangers or in case the effect is known it could be used in a beneficial way. In the present research effortwe want to study the effect of strongly changing thermodynamic properties on the turbulent flow and heat transfer in a wall bounded flow, with a specific focus on the local effect of buoyancy forces, flow laminarization and deterioration or enhancement of heat transfer. In this short paper we will discuss the numerical model and give only a few results for developing turbulent pipe flow.

\section{Governing Equations and Numerical Model}

As starting point for the modeling of supercritical flows we will use the fully compressible Navier-Stokes equations and simplify these equations for low Mach numbers. The compressible Navier Stokes equations read (see, e.g. [4])

$$
\begin{aligned}
& \frac{\partial \rho}{\partial t}=-\frac{\partial \rho u_{i}}{\partial x_{i}} \\
& \frac{\partial \rho u_{i}}{\partial t}=-\frac{\partial \rho u_{i} u_{j}}{\partial x_{i}}-\frac{\partial p}{\partial x_{i}}+\frac{\partial \tau_{i j}}{\partial x_{i}}-\rho g \\
& \frac{\partial \rho h}{\partial t}=-\frac{\partial \rho u_{i} h}{\partial x_{i}}-\frac{\partial q}{\partial x_{i}}+\tau_{i j} \frac{\partial u_{i}}{\partial x_{i}}+\frac{\partial p}{\partial t}+u_{i} \frac{\partial p}{\partial x_{i}} \\
& q=-\kappa \frac{\partial T}{\partial x_{i}} ; \tau_{i j}=\mu\left(\frac{\partial u_{i}}{\partial x_{j}}+\frac{\partial u_{j}}{\partial x_{i}}-\frac{2}{3} \frac{\partial u_{k}}{\partial x_{k}}\right)
\end{aligned}
$$

In these equations $\rho$ denotes the fluids density, $u_{i}$ the fluids velocity vector, $p$ the pressure, $\tau_{i j}$ the Newtonian stress tensor, $h$ the enthalpy, $q$ the heat flux, $\kappa$ the thermal conductivity, $T$ the temperature and $\mu$ the viscosity. In most supercritical heat transfer cases the fluid velocity is small compared the speed of sound, i.e. the Mach number is small. This observation can be used to simplify the governing equations. First the governing equations are made non dimensional with help of the following non-dimensional variables indicated with an asterisk:

$$
\begin{gathered}
t^{*}=\frac{t L}{U}, x^{*}=\frac{x}{L}, \rho^{*}=\frac{\rho}{\rho_{\infty}}, u_{i}^{*}=\frac{u_{i}}{U}, p^{*}=\frac{p}{\rho_{\infty} c^{2}}, T^{*}=\frac{T c_{p, \infty}}{c^{2}}, h^{*}=\frac{h}{c^{2}}, \mu^{*}=\frac{\mu}{\mu_{\infty}}, \kappa^{*}=\frac{\kappa}{\kappa_{\infty}} \\
M a=\frac{U}{c}, \operatorname{Re}=\frac{\rho_{\infty} U L}{\mu_{\infty}}, \operatorname{Pr}=\frac{c_{p, \infty} \mu_{\infty}}{\kappa_{\infty}}, F r=\frac{U}{\sqrt{g L}}
\end{gathered}
$$


Where $L, U$ are characteristic length and velocity scale, the subscript $\infty$ denotes reference values of the properties, $c_{p}$ is the heat capacity at constant pressure and $c$ is the speed of sound. Note that the velocity is scaled with a characteristic fluid velocity scale, $U$, and the pressure, temperature and enthalpy (the thermodynamic variables) are scaled with the acoustic velocity scale $c$. Where it should be noted that it is assumed that $U \square c$. Applying these scaling parameters to the momentum equations gives:

$$
\begin{aligned}
& \frac{\rho_{\infty} U}{L} \frac{\partial \rho^{*}}{\partial t^{*}}=-\frac{\rho_{\infty} U}{L} \frac{\partial \rho^{*} u_{i}^{*}}{\partial x_{i}^{*}} \\
& \frac{\rho_{\infty} U^{2}}{L} \frac{\partial \rho^{*} u_{i}^{*}}{\partial t^{*}}=-\frac{\rho_{\infty} U^{2}}{L} \frac{\partial \rho^{*} u_{i}^{*} u_{j}^{*}}{\partial x_{i}^{*}}-\frac{\rho_{\infty} c^{2}}{L} \frac{\partial p^{*}}{\partial x_{i}^{*}}+\frac{\mu_{\infty} U}{L^{2}} \frac{\partial \tau_{i j}^{*}}{\partial x_{i}^{*}}-\rho^{*} \rho_{\infty} g \\
& \frac{\rho_{\infty} U c^{2}}{L} \frac{\partial \rho^{*} h^{*}}{\partial t_{*}}=-\frac{\rho_{\infty} c^{2} U}{L} \frac{\partial \rho^{*} u_{i}^{*} h^{*}}{\partial x_{i}^{*}}-\frac{c^{2} \kappa_{\infty}}{c_{p} L^{2}} \frac{\partial q^{*}}{\partial x_{i}^{*}}+\frac{\mu_{\infty} U^{2}}{L^{2}} \tau_{i j}^{*} \frac{\partial u_{i}^{*}}{\partial x_{i}^{*}}+\frac{\rho_{\infty} U c^{2}}{L}\left(\frac{\partial p^{*}}{\partial t^{*}}+u_{i}^{*} \frac{\partial p^{*}}{\partial x_{i}^{*}}\right) \\
& \frac{\partial \rho^{*}}{\partial t^{*}}=-\frac{\partial \rho^{*} u_{i}^{*}}{\partial x_{i}^{*}} \\
& \frac{\partial \rho^{*} u_{i}^{*}}{\partial t^{*}}+\frac{\partial \rho^{*} u_{i}^{*} u_{j}^{*}}{\partial x_{i}^{*}}=-\frac{1}{M a^{2}} \frac{\partial p^{*}}{\partial x_{i}^{*}}+\frac{1}{\operatorname{Re}} \frac{\partial \tau_{i j}^{*}}{\partial x_{i}^{*}}-\frac{\rho^{*}}{F r^{2}} \\
& \frac{\partial \rho^{*} h^{*}}{\partial t^{*}}=-\frac{\partial \rho^{*} u_{i}^{*} h^{*}}{\partial x_{i}^{*}}-\frac{1}{\operatorname{Re} \operatorname{Pr}} \frac{\partial q^{*}}{\partial x_{i}^{*}}+\frac{M a^{2}}{\operatorname{Re}} \tau_{i j}^{*} \frac{\partial u_{i}^{*}}{\partial x_{i}^{*}}+\left(\frac{\partial p^{*}}{\partial t^{*}}+u_{i}^{*} \frac{\partial p^{*}}{\partial x_{i}^{*}}\right)
\end{aligned}
$$

At this stage it will be useful to introduce the following series expansion for the pressure in terms of the Mach number, which is assumed to be small $(\mathrm{Ma}<<1)$ :

$$
p^{*}=p_{0}^{*}+(M a)^{1} p_{1}^{*}+(M a)^{2} p_{2}^{*}+\ldots
$$

From the momentum equation it now follows that $p_{0}^{*}$ is spatially constant and the value of the constant depends only on time. The pressure $p_{2}^{*}$ balances the velocity fluctuations. $p_{0}^{*}$ can be interpreted as the thermodynamic pressure and $p_{2}^{*}$ as the hydrodynamic pressure. With this observations the last term in the energy equation (the term between brackets) is order $M a^{2}$ and the energy equation reduces to a simple transport equation for the enthalpy. The hydrodynamic pressure depends now only on the velocity field and becomes and elliptic quantity. Therefore we can solve the equations as an incompressible fluid system. The thermodynamic properties like viscosity, heat capacity, density and thermal conductivity follow from a thermodynamic relation, e,g [3], $\mu=\mu(p, h)$ which in the case of constant thermodynamic pressure simply reduces to $\mu=\mu\left(p_{0}, T\right)$.

The equations above or discretized on a three dimensional staggered grid, with scalar quantities the cell centers and the velocity components at the cell faces, see [5]. The spatial derivatives in the momentum equations are approximated with $2^{\text {nd }}$ order central differences or $6^{\text {th }}$ order compact finite differences.. Time advancement is done with the pressure correction method. To stabilize the calculations for large density gradients we use the predictor corrector method of [6]. The thermodynamic relations are computationally very expensive. Therefore first polynomial functions are generated from the thermodynamic relations over the range of interest. Subsequently the polynomials are used for the runtime evaluation of the thermodynamic properties. 


\section{Flow Domain and Some Results}

The flow geometry we study is sketched in Figure 3. Isothermal incompressible turbulent flow with a temperature slightly below the pseudo-critical temperature is generated in a small cylindrical domain with periodic in and outflow conditions. The isothermal flow is fed into a domain with constant wall heating. The temperature of the fluid will slowly increase and at a certain point the flow will reach the pseudo critical temperature. The Reynolds number based on the bulk velocity at the inlet of the developing pipe is equal to 5300 the total number of grid points is approximately 20 million. In Figure 4 we show a contour plot of the so-called Q-criterion at critical conditions and subcritical conditions. Cleary the property variations have a very large effect on the turbulence and the associated heat transfer.

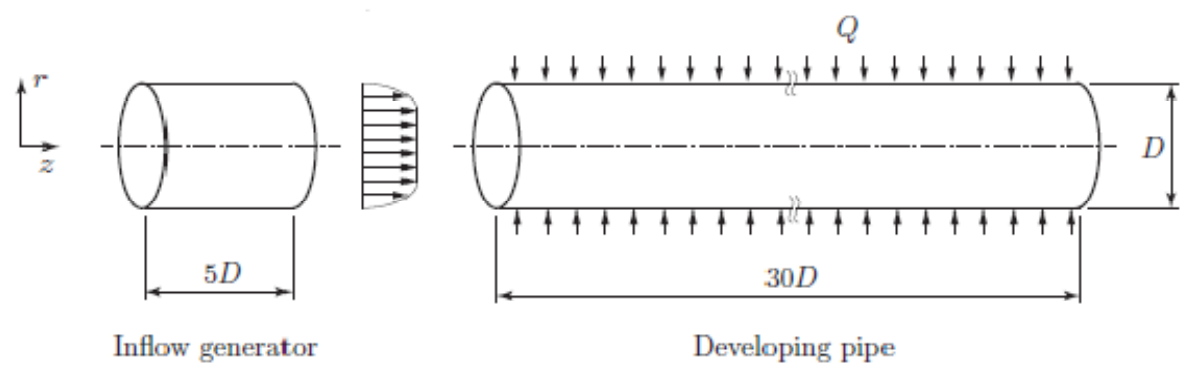

FIGURE 3. A sketch of the flow geometry. Inflow conditions for the developing pipe section are generated by the inflow generator in which we solve the isothermal 3D Navier Stokes equations with periodic boundary conditions.
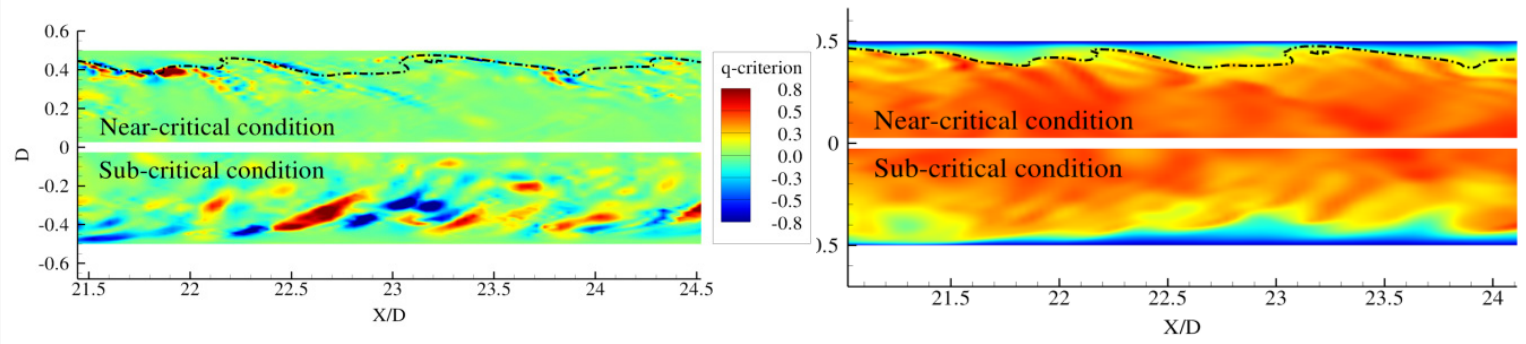

FIGURE 4. Turbulent pipe flow simulation for the case with constant (lower part) and strongly varying properties (upper part), along the dashed line the temperature is equal to the pseudo-critical temperature. Left: The Q-criterion (positive values correspond to vorticity dominated regions while negative values correspond to shear dominated regions. Right the streamwize velocity in the pipe.

\section{REFERENCES}

1. A.B. Kogan\& H. Meyer, 2001, Heat transfer and convection onset in a compressible fluid 3 He near the critical point, Phys. Rev. E. 63, 056310.

2. S.M. Liao \& T.S. Zhao, 2002, An experimental investigation of convective heat transfer to supercritical carbon dioxide in miniature tubes, Int. J. of Heat and Mass Transfer, 45, 5025-5034.

3. P. Colonna and T. P. van der Stelt, "FluidProp: A program for the estimation of thermophysical properties of fluids." software, www.fluidprop.com, 2004-2011.

4. R.B. Bird, W.E. Stewart, \& E.N. Lightfoot, 1960. Transport phenomena, Wiley.

5. B.J. Boersma, 2011, A $6^{\text {th }}$ order staggered compact finite difference method for the incompressible Navier-Stokes and scalar transport equations, J. of Comp. Phys, 230, 4940-4954.

6. H.N. Najm. P.S. Wyckoff \& O.M. Knio, 1998, A semi-implicit numerical scheme for reacting flow: I stiff chemistry, J. of Comp. Phys, 143, 381-402. 